\title{
Relaxation of photovoltage in ITO-Ge-Si heterojunction with Ge nanostructured thin films
}

\author{
S.A. Iliash ${ }^{1}$, Yu.V. Hyrka ${ }^{1}$, S.V. Kondratenko ${ }^{1}$, V.S. Lysenko ${ }^{2}$, Yu.M. Kozyrev ${ }^{3}$, V.V. Lendel ${ }^{1}$ \\ ${ }^{1}$ Taras Shevchenko National University of Kyiv, 64/13, Volodymyrs'ka str., 01601 Kyiv, Ukraine \\ E-mail: iliashsviatoslav@gmail.com \\ ${ }^{2}$ Institute of Semiconductor Physics, 41, prospect Nauky, 03680 Kyiv, Ukraine \\ ${ }^{3}$ O.O. Chuiko Institute of Surface Chemistry, 17, Generala Naumova str., 03164 Kyiv, Ukraine
}

\begin{abstract}
The paper focuses on experimental study of the photovoltage time decay in ITO-Ge-Si heterojunction with Ge nanostructured thin film. Kinetics under $650 \mathrm{~nm}$ excitation within the temperature range 80 to $290 \mathrm{~K}$ are successfully described by a single exponential function with temperature-dependent decay constants. Photovoltage relaxation is modeled taking into account the hopping nature of electron transport in the band of localized states.
\end{abstract}

Keywords: heterostructure, ITO-Ge-Si heterojunction, thin films, photovoltage, transient voltage.

Manuscript received 13.01.17; revised version received 27.04.17; accepted for publication 14.06.17; published online 18.07.17.

\section{Introduction}

Semiconductor nanoclusters (NCs) attract interest due to new possibilities for many electronic applications, e.g., field-effect transistors, IR photodetectors, and solar cells [1-3]. The improvement in the device performance is reached by decreasing the distance between the NCs, narrowing the dispersion of $\mathrm{NC}$ sizes as well as changing the NCs capping material.

The nanocrystalline Ge films grown on silicon substrates are of particular interest, due to their compatibility with silicon technology. Electronic spectrum, charge carrier transport and optical properties of SiGe-based nanostructures essentially depend on the mechanical stress caused by mismatch of parameters of $\mathrm{Si}$ and Ge crystal lattices as well as variations in the size and composition of $\mathrm{Ge}$ nanostructures. This makes possibilities for creating thin films with predetermined properties for applications in optoelectronics, photovoltaics and sensors.

\section{Experimental details}

The molecular beam epitaxy (MBE) technique was used to prepare monolayer and multilayer $\mathrm{Ge}-\mathrm{Si}(100)$ $\mathrm{NC}$ arrays with the clusters of various sizes and surface density. The (100) oriented plates of $p$-Si with resistivity of $7.5 \Omega \cdot \mathrm{cm}$ and diameter of $76 \mathrm{~mm}$ were used as substrates. The background pressure of residual gases in the MBE set-up was $6 \cdot 10^{-10}$ Torr. After desorption of the passivating $\mathrm{Si}$ oxide film from the $\mathrm{Si}$ substrate, which was exposed to a Si beam of a weak intensity and kept at $800{ }^{\circ} \mathrm{C}$ in the course of this process, a buffer Si film, about 0.1 to $0.5 \mu \mathrm{m}$ thick, was deposited onto the $\mathrm{Si}$ surface. This film produces a high-contrast $\operatorname{Si}(100) 2 \times 1$ RHEED pattern typical of clean $\mathrm{Si}$. The Ge nanocrystalline layers were deposited at $350{ }^{\circ} \mathrm{C}$. The deposition rate was $0.15 \mathrm{~nm} / \mathrm{min}$ for $\mathrm{Si}$ and $5.0 \mathrm{~nm} / \mathrm{min}$ for $\mathrm{Ge}$.

The ITO films $\left(95 \% \mathrm{In}_{2} \mathrm{O}_{3}+5 \% \mathrm{SnO}_{2}\right)$ with a thickness of $50 \mathrm{~nm}$ were deposited on the $p$-Si(001) boron-doped substrate or $\mathrm{Ge}$ thin film by vacuum magnetron sputtering at $125^{\circ} \mathrm{C}$.

AFM measurements were performed with an NTMDT Ntegra microscope using cantilevers with a tip radius of about $10 \mathrm{~nm}$. Fig. 1a shows the AFM images of the surface consisting of the nanocrystalline Ge film.

The photovoltage temporal dependences were recorded on a Siglent $70-\mathrm{MHz}$-bandwidth and $1 \mathrm{M} \Omega$ input impedance digital oscilloscope with a low-noise amplifier (AD8138) that measured a voltage signal drop across the series load resistance of $47 \mathrm{k} \Omega$ (see Fig. 1b). 


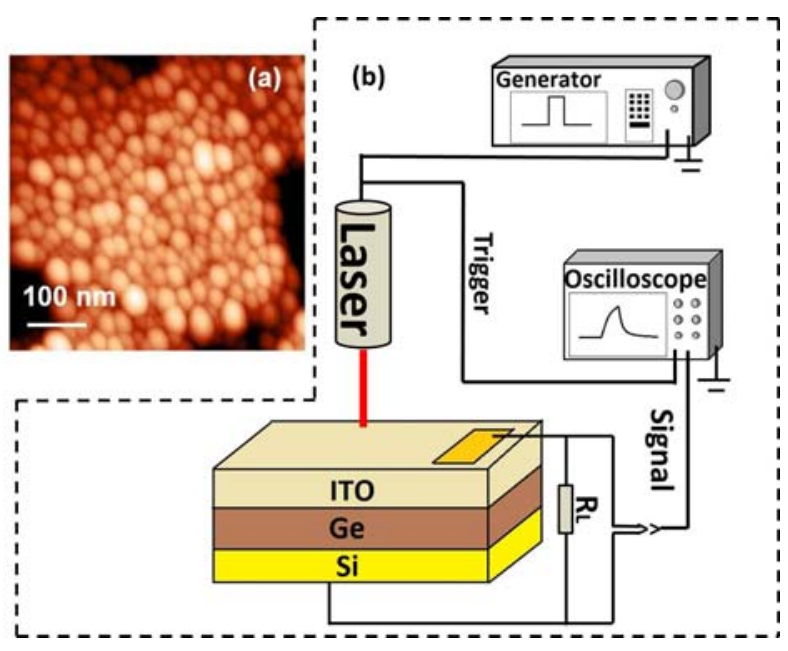

Fig. 1. AFM images of ITO-Ge-Si heterojunction surface (a). The scheme for transient photocurrent measurements (b).

\section{Results and discussion}

Morphology studies showed that low-temperature $\mathrm{Ge}$ epitaxy on $\mathrm{Si}(001)$ surface at $350^{\circ} \mathrm{C}$ and high (about $5 \mathrm{~nm} / \mathrm{min}$ ) epitaxy rate leads to formation of nanocrystalline films consisting of Ge crystallites, 5-6 $\mathrm{nm}$ in size, which are pseudomorphic to the substrate.

Fig. 2 shows the dark current-voltage $(I-V)$ curves of ITO-Ge-pSi and ITO-pSi structures at room temperature. The current in the reverse bias is the order of magnitude lower than in forward bias due to the potential barrier of ITO-Si heterojunction.

The shape of curves was analyzed within the framework of the standard diode equation. If the diffusion current and the recombination one are both present in a single diode, the diode model usually describes the dark $I-V$ dependences. The current density $J$ is described as follows:

$J(V)=J_{0}\left(\exp \left(\frac{e\left(V-I R_{S}\right)}{n k T}\right)-1\right)+\frac{V-I R_{S}}{R_{S H}}$,

where $J_{0}$ is the diode reverse saturation current, $k-$ Boltzmann's constant, $T$ - absolute temperature, $R_{S}$ and $R_{S H}$ are the series and shunt resistances, respectively. The diode ideality factor is a characteristic of the generation-recombination (G-R) processes, which dominate in the $p-i-n$ diodes. They are mainly considered as active only in the space-charge region. It is commonly accepted that $n=1$, when the diffusion current dominates, and $n=2$, when the recombination current is dominant. Analysis of the forward bias shows that the value $n \approx 3$ within the range from 0.2 to $0.4 \mathrm{~V}$ due to significant impact of recombination in the space charge heterojunction and limiting forward current by series resistance. Under $U>0.4 \mathrm{~V}$, the series resistance has a substantial effect on the value of current, which is found to be 193.4 and $204.9 \Omega \cdot \mathrm{cm}^{2}$ at $290 \mathrm{~K}$ for ITO-Ge$p \mathrm{Si}$ and ITO-pSi structures, respectively. The inset to
Fig. 2 shows the $I-V$ dependences measured under AM1.5G illumination. The structure of Ge thin films reveales higher values of short circuit current and photoEMF in comparsion with the reference ITO-pSi structure due to accumulation of a positive charge by Ge NCs and lower values of $R_{S}$.

Fig. 3 shows the photovoltage temporal dependences for ITO-Ge-pSi and ITO-pSi structures measured after excitation by the laser diode with the wavelength $650 \mathrm{~nm}$ at different temperatures. Decay curves are approximated by exponential dependence within the investigated temperature range 80 to $290 \mathrm{~K}$ :

$U(t) \sim \exp (-t / \tau)$

where $\tau$ is a decay constant.

Fig. 4 shows temperature dependences of photovoltage decay constants $\tau(T)$ for ITO-Ge- $p \mathrm{Si}$ heterojunction and reference ITO-pSi structure. The structure with Ge nanostructured thin film reveales faster relaxation of photo-EMF within the temperature range 80 to $290 \mathrm{~K}$. The reason for this behavior is that Ge nanoclusters are additional recombination centers.

The rate of recombination of nonequilibrium carriers involving Ge states determines the value $\tau$ for the structure ITO-Ge-pSi. The decay constant slightly increases from 28 up to $33 \mathrm{~ms}$ under the temperature decrease from 290 down to $220 \mathrm{~K}$. However, the value $\tau$ rises with decreasing temperature in the range $T<220 \mathrm{~K}$, which is described as follows:

$$
\tau(T) \sim \exp \left(-T / T_{0}\right)
$$

where $T_{0}$ is the constant equal to $90.2 \mathrm{~K}\left(k T_{0}=7.8 \mathrm{meV}\right)$ for structures with Ge nanoclasters.

The observed temperature dependence can be explained by the peculiarities of photogenerated charge carrier transport in a Ge thin film.

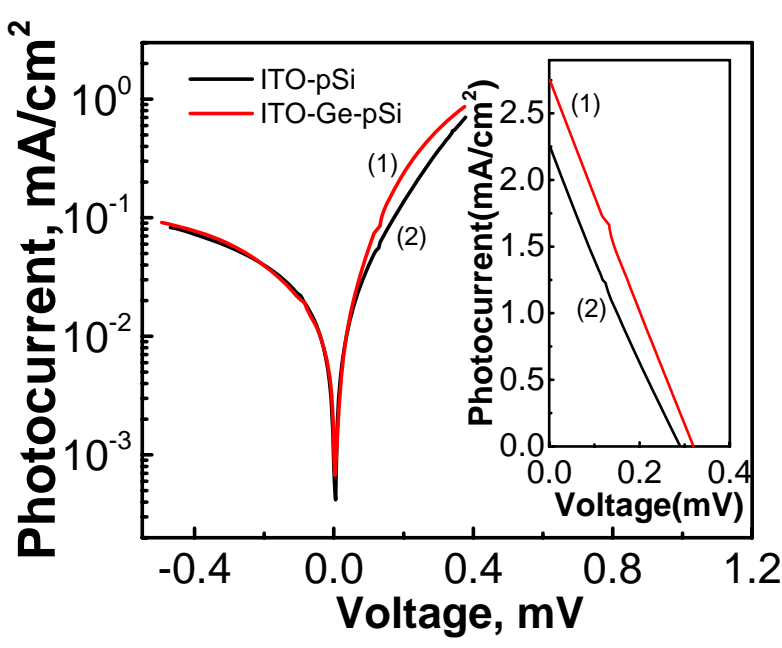

Fig. 2. Dark $I-V$ curves of the ITO-Ge-pSi (1) and ITO-pSi (2) structures. The inset shows the $I-V$ dependences measured under AM1.5G illumination at $290 \mathrm{~K}$. 


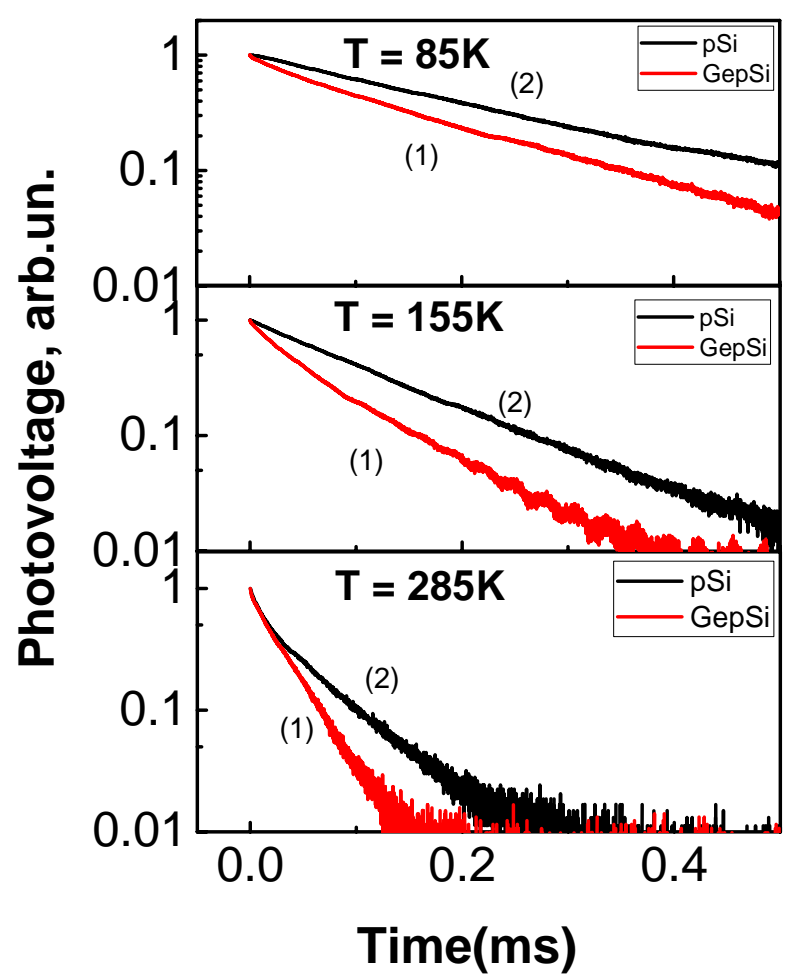

Fig. 3. Time dependences of photovoltage decay for the ITOGe-pSi (1) and ITO-pSi (2) structures, measured at different temperatures: 85,155 and $285 \mathrm{~K}$.

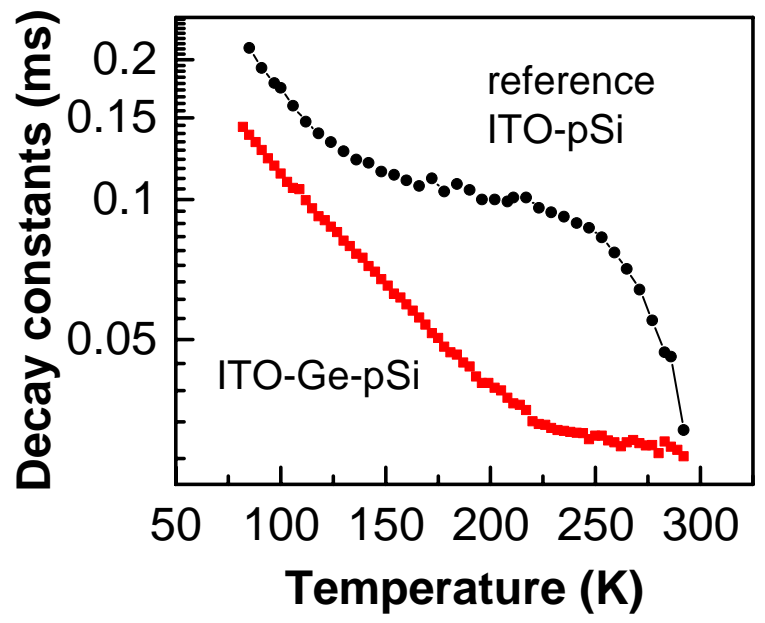

Fig. 4. The temperature dependence of photovoltage decay constants for the ITO-Ge-pSi heterojunction and reference ITO-pSi structure.

The charge transport is defined by thermal activation to the band of extended states (multiple trapping) or by hopping via localized states in the film consisting of crystalline Ge NCs of $5 \ldots 6 \mathrm{~nm}$ in size, where the space between them is filled with air and contains amorphous phase of Ge. Under high temperatures, $T>220 \mathrm{~K}$, the recombination rate is determined by times of electron capture/release from traps. When temperature becomes lower, the Ge NCs becomes a giant trap for holes accumulating a positive charge causing downward band banding in the underlying $\mathrm{Si}[4,5]$. On the basis of the foregoing, we assume that holes trapped by Ge are practically immobile. Electrons have a chance to meet a hole and recombine only during their transport. In this case, recombination rate depends on the total density of holes and mobility of photoexcited electrons. Under low temperatures, electron transport occurs by hopping within the band of localized states and recombination occurs between hopping electrons and holes trapped by Ge NCs. The number of trapped holes increases under the temperature decrease, while the average jump distance of electrons becomes higher. The photoelectrons will be more and more accumulated in more isolated states with longer release time constants, which will increase $\tau$ exponentially with decreasing temperature [6].

\section{Conclusions}

The obtained results indicate that the $\mathrm{Ge}$ film significantly affects the transport and recombination of nonequilibrium carriers in barrier structures based on heterojunction ITO-pSi. The increase in the short circuit current value and photo-EMF in the structure of the Ge thin film barrier is caused by a higher value as compared with the reference ITO-pSi heterojunction. The lifetime of photogenerated carrier is determined by the recombination channel efficiency, which is associated with Ge NCs. The hopping transport of photoexcited electrons in $\mathrm{Ge}$ thin films determines the recombination rate in ITO-Ge-pSi heterostructures.

\section{References}

1. Brunner K. Si/Ge nanostructures. Rep. Prog. Phys. 2002. 65. P. 27.

2. Liu J., Beals M., Pomerene A., Bernardis S., Sun R., Cheng J., Kimerling L.C. \& Michel J. Waveguide-integrated, ultralow-energy GeSi electro-absorption modulators. Nat. Photonics. 2008. 2. P. 433-437.

3. Cosentino S., Liu P., Le S.T. et al. High-efficiency silicon-compatible photodetectors based on $\mathrm{Ge}$ quantum dots. Appl. Phys. Lett. 2011. 98. P. 221107.

4. Lysenko V.S., Gomeniuk Y.V., Kudina V.N. et al. Hopping conduction and LF noise in structures with $\mathrm{Ge}$ nanoclusters grown on oxidized $\mathrm{Si}$ (001). J. Mater. Sci. 2016. 51, No. 19. P. 8799-8811.

5. Garbar N.P., Kudina V.N., Lysenko V.S., Kondratenko S.V. \& Kozyrev Yu.N. Effect of Genano-islands on the low-frequency noise in $\mathrm{Si} / \mathrm{SiO}_{\mathrm{x}} / \mathrm{Ge}$ structures. Adv. Mater. Res. 2014. 854. P. 21-27.

6. Iacchetti A., Natali D., Binda M., Beverina L. \& Sampietro M. Hopping photoconductivity in an exponential density of states. Appl. Phys. Lett. 2012. 101, No. 10. P. 103307. 